\title{
On the Combined Use of Ground Penetrating Radar and Crack Meter Sensors for Structural Monitoring: Application to the Historical Consoli Palace in Gubbio, Italy
}

\author{
G. Ludeno ${ }^{1} \mathbb{D} \cdot$ N. Cavalagli ${ }^{2} \cdot$ F. Ubertini $^{2} \cdot$ F. Soldovieri $^{1} \cdot$ I. Catapano $^{1}$
}

Received: 9 October 2018 / Accepted: 20 March 2019 / Published online: 6 April 2019

(c) The Author(s) 2019

\begin{abstract}
The paper deals with joint use of non-invasive monitoring technologies and civil engineering analysis methods aimed at providing multi-sensing information about the structural health of historical and cultural assets. Specifically, linear variable displacement transducers (LVDT) and ground penetrating radar (GPR) are considered for monitoring a significant crack affecting the Consoli Palace in Gubbio, Italy, precisely one of the walls of the cross-hall leading to the Loggia. In this frame, LVDT is adopted to control horizontal amplitude variations of the crack, while GPR is applied to investigate the wall interior and to detect the occurrence of inner issues related to the visible appearance of the crack on the wall surface. The effectiveness of GPR surveys is improved by means of a microwave tomography-based data processing strategy. The main result is that there is a consistency between the monitoring outputs of LVDT, which allowed us to display the crack widening/contraction due to the seasonal temperature variations, and the fact that no significant changes of the geometry of the inner areas of the walls were observed by the GPR.
\end{abstract}

Keywords Cultural heritage $\cdot$ Structural monitoring $\cdot$ Linear variable displacement transducers · Ground Penetrating radar · Microwave imaging · Technologies integration

G. Ludeno

ludeno.g@irea.cnr.it

N. Cavalagli

nicola.cavalagli@unipg.it

F. Ubertini

filippo.ubertini@unipg.it

F. Soldovieri

soldovieri.f@irea.cnr.it

I. Catapano

catapano.i@irea.cnr.it

1 Institute for Electromagnetic Sensing of the Environment, National Research Council of Italy, Via Diocleziano 328 - 80124, Naples, Italy

2 Department of Civil and Environmental Engineering, University of Perugia, Via Goffredo Duranti, 93, 06125 Perugia, Italy 


\section{Introduction}

Diagnostics and monitoring of the health status of manmade structures, such as bridges, dams, public and private buildings as well as monuments, are a significant issue and involve continuous research activities. The aim is the development of effective sensing/ observational strategies capable of improving maintenance and intervention also by looking to their economic sustainability (Chong et al. 2001; Wang et al. 2014; Chen et al. 2017).

In this frame, currently there is a huge interest towards the joint and cooperative use of state of art civil engineering analysis methods and electromagnetic sensing techniques, with the aim to provide objective data suitable to support and integrate information gathered by means of ordinary visual inspections. This appears, indeed, as a key issue in order to effectively plan the maintenance activities and to extend the structure lifespan (ASCE 2013; Deutscher Bundestag 2014; Eastman et al. 2008; Sankarasrinivasan et al. 2015). Moreover, integration of data and information coming from an advanced structural health monitoring (SHM) system, involving different types of sensing technologies with building information modelling, is a valuable tool to enhance the effectiveness of manmade structure management. Such an integration should enable models, built in the design or assessment phase, to be able to "react" to changes occurring over the life cycle of a structure, as discussed in Rio et al. (2013), Chen et al. (2014) and Sternal and Dragos (2016).

A review of needs and opportunities of the integration of sensing technologies, including civil engineering analysis methods, is given in Masini and Soldovieri (2017), which presents the general framework of a systematic approach exploiting the integration of sensing and observational technologies. This integration represents a technological tool to enable the monitoring not only of the asset but also of the embedding territory. Therefore, context analysis and site health monitoring chains are capable of providing an always-updated situational awareness of the asset and the territory, in view of a sustainable management and protection of cultural heritage $(\mathrm{CH})$. In fact, an always-updated situational awareness is a crucial information in designing and implementing reliable cost-effective long-term maintenance actions and operational procedures for risk management and allows us to:

1. Prioritize, define and optimize the maintenance interventions by also looking to the economic sustainability and by accounting for the fragility of cultural heritage and reversibility of the interventions;

2. Improve risk management through a clear identification of risks in terms of magnitude and spatial location;

3. Enable a reliable diagnosis by providing detailed information about the historical and technological contexts of heritage materials and objects.

A review concerning the integration of sensing technologies for $\mathrm{CH}$ diagnosis and monitoring is given in Masini and Soldovieri (2011), where several case studies demonstrate the capabilities of the integrated approaches for the study and conservation of $\mathrm{CH}$. Another valuable review is given in Baeza et al. (2018), which presents several examples about the use of SHM systems for inspecting Spanish historic constructions and civil engineering facilities. In this frame, several non-destructive testing techniques, among which are thermography, ground penetrating radar (GPR), synthetic-aperture radar (SAR), ultrasound, vibration-based methods and linear variable displacement transducer (LVDT), are taken into account and their main applications are summarized. 
Further examples are given in Orban and Gutermann (2009), Proto et al. (2010), Kilic (2015) and Masini et al. (2017). In Orban and Gutermann (2009), an overview of nondestructive, minimally destructive and monitoring methods is given and the efficacy of the considered methods is discussed by presenting results of a testing programme, which was aimed at developing technical recommendations for assessment, inspection and maintenance of masonry arch railway bridges. In Proto et al. (2010), an example referred to an integrated observational chain, where satellite, aerial and in situ technologies are combined with civil engineering analysis methods is presented. This example concerns the investigation of the Basento viaduct in Potenza (Southern Italy), which is one of the most important architectural modern constructions in Italy, and it is considered an example of modern $\mathrm{CH}$ asset. In Kilic (2015), an integrated approach combining visual inspection, GPR and infrared thermography is presented as an effective tool to gather information on both visible and hidden defects, such as cracks, affecting the structural condition of historical buildings. In Masini et al. (2017), in situ non-invasive sensing techniques, specifically GPR, seismic tomography and infrared thermography (IRT), are deployed for the diagnosis of conservation and the restoration state of structures and surfaces of archaeological monuments at Regio VIII in Pompeii. This study also provided indication for successive restoration works.

The aforementioned works are only few examples of a very large existing literature on SHM systems applied to architectural heritage and historic structures. Indeed, it is worth noting that, in the last 10 years, research activities and technological developments have constantly faced the optimization and integration of electromagnetic sensing technologies and civil engineering methods. Further valuable contributions on this topic are in Sansoni et al. (2009), Burrows et al. (2007) and Masini et al. (2012).

As a further contribution to the above, for the wide and complex topic concerning the joint use of different sensing technologies, this paper presents a case study, wherein LVDT and GPR are deployed cooperatively at the Consoli Palace, Gubbio, Italy. Conosli Palace is an important Italian historical building characterized by an articulated distribution of volumes. It is well known that historical masonry constructions are often affected by physiological structural pathologies, which often result into relative movements between structural elements generating cracks. This aspect is mainly due to the inability of the material to accumulate large deformations and/or to withstand tensile stresses. With the exception of damages related to strong unexpected events, such as earthquakes, the crack patterns are generally characterized by slow changes over time. Nevertheless, the continuous monitoring of these cracking phenomena is very useful to understand the evolution of possible mechanisms associated with a damage scenario.

As it is well known, LVDTs are state of art displacement sensors whose use in civil engineering structures is widespread (Joshi and Harle 2017; Noel et al. 2017) and typically aimed at monitoring the evolution of cracks related to structural pathologies, causing relative movements among different parts of the same building. In this perspective, LVDT are typically exploited in "static" monitoring systems by performing measurements with relatively low sampling frequencies (on the order of a sample every few seconds). Conversely, in "dynamic" monitoring systems, structural response is monitored at large sampling frequencies (on the order of tens of $\mathrm{Hz}$ ) in order to characterize structural vibrations in terms of accelerations, velocities or displacements (Cabboi et al. 2017; Oliveira et al. 2018; Pierdicca et al. 2018; Roselli et al. 2018; Ubertini et al. 2018). Moreover, LVDTs are usually installed across major cracks in order to check their opening/closing trends over time and highlight anomalous situations and several examples can be found in the literature (Hejll et al. 2006; Gioffré et al. 2008; Marazzi et al. 2011; Frangopol et al. 2012; Coïsson 
and Blasi 2015; Coïsson and Ottoni 2015; Ottoni and Blasi 2015; Rossi and Rossi 2015; Duvnjak et al. 2016; Masciotta et al. 2016; Sánchez et al. 2016; Cigada et al. 2017; Blanco et al. 2018). The majority of these works highlights the ease of installation and measurement accuracy of LVDT sensors, while also reporting that, in long-term static monitoring applications, measurements contain the effects of changing environmental conditions, mainly temperature and humidity, causing material deformation. In order to take into account these effects, temperature and/or humidity sensors are often applied near the transducers (Lorenzoni et al. 2013; Masciotta et al. 2017; Saisi et al. 2018; Kita et al. 2019). The simultaneous recording of crack amplitudes and environmental parameters is key to remove environmental effects from the displacement data and to analyse the residuals associated with purely structural movements. Moreover, the correlation analysis between opening/closing of a crack and the environmental data allows improving the knowledge of the construction behaviour and, in some cases, of the mechanisms associated with the damage scenario (Russo 2013; Lombillo et al. 2016; Lorenzoni et al. 2016; Ceravolo et al. 2017).

On the other hand, as far as GPR is concerned, it is worth remembering that it is a geophysical method, commonly used for archaeological and civil engineering surveys, which allows subsurface prospection aimed at detecting and localizing hidden objects and anomalies (Conyers 2013; Goodman and Piro 2013). Moreover, in the frame of structural assessment, GPR is a proper tool to gather information about structural elements, such as the presence of inner reinforcement elements and material layers (Krysinski and Hugenschmidt 2015; Diamanti et al. 2017; Pérez et al. 2018). In addition, GPR allows the imaging of cracks, voids and inner anomalies, which might endanger the conservation and the integrity of the structure (Solla et al. 2011; Catapano et al. 2017; Masini and Soldovieri 2017). Furthermore, GPR allows a check of the effectiveness of previous restoration works (Masini et al. 2010; Leucci et al. 2011).

According to the above considerations, LVDT and GPR are on-site technologies worthy to be included in a SHM system devoted to improve efficiency, versatility and ability of the maintenance process of $\mathrm{CH}$ assets, while also accounting for the accomplishment of requirements and constraints arising in a specific application context. The combined use of these different technologies becomes significant in the cases of blind cracks, for which the LVDT gives information about the structural behaviour resulting on the external side of the wall with high precision, while GPR improves the knowledge of the inside of the structure in the area affected by the crack. This statement is, herein, assessed by presenting results referred to the monitoring of a cracking phenomenon visible on a wall of the crosshall conducting to the Loggia of the Consoli Palace. Such a monitoring study is part of a wider analysis carried out in the frame of the H2020 HEritage Resilience Against CLimate Events on Site (HERACLES) project, whose main goal is the design of, validation and promotion of responsive systems/solutions for effective resilience of $\mathrm{CH}$ against climatechange effects (http://www.heracles-project.eu/). Within this general frame, LVDT and GPR have been considered for the specific aim of gathering information useful to define the damage status of the Consoli Palace. Specifically, LVDT is deployed to monitor the crack amplitude variations occurring in 1 year, while two GPR surveys, performed at the beginning and at the end of the monitoring period, are exploited to investigate the wall interior and to detect the possible occurrence of inner changes related to the visible appearance of the crack on the wall surface.

The paper is organized as follows. Section 2 summarizes the working principles of the considered technologies and briefly reviews examples on their use for structural assessment. Section 3 describes the case study, while LVDT monitoring and GPR surveys results are presented in Sect. 4 and discussed in Sect. 5. Conclusions end the paper. 


\section{Monitoring Technologies}

\subsection{LVDT for the Static Monitoring of Structures}

The LVDT acronym is used in the literature with two similar but distinct meanings. The former refers to its working task as an electro-mechanical device (linear variable differential transformer), while the latter to its direct use as a transducer (linear variable displacement transducer).

The sensor consists of a central core in motion into a cylindrical device hosting a system of coils, which transforms a mechanical displacement of the core into an electrical voltage signal. Hence, its working principle is the mutual induction. A primary coil, positioned in the central part of the cylinder and supplied with an alternating current, generates a magnetic field within the cylindrical device, where the core is inserted. Two secondary coils, having the same number of windings but with opposite polarities, are installed at the extreme sides of the cylinder, near the primary ones. When the core moves between the coils, the two parts of the secondary coil receive different voltages, due to the different mutual inductances. Figure 1 shows a sketch of the LVDT working principle. For more details, the interested readers can refer to the literature both for standard systems (Zumbahlen 2008; Zhang 2010; Williams 2011; Bolton 2015; Pei 2015; Morris and Langari 2012) and for new advanced solutions (Knudson and Rempe 2012; Mandal et al. 2018; Mondal et al. 2018).

With its the quite apparent simplicity, an LVDT is able to provide, with a very high precision, the displacement variation between two fixed points, within a range of millimetres or, at most, some centimetres. For this reason, it is often considered as a very useful device in the framework of structural monitoring, giving the possibility to continuously check, with high precision, the stability of a crack or a junction allowing relative movements between two structural members (e.g., bridge bearing). In spite of this level of information accuracy, the installation requires a high level of care and attention, especially in the case of historical constructions. Since the LVDT provides displacement information between only two points and along a predefined direction, the local behaviour obtained by the sensor should be, as much as possible, representative of the global behaviour of the object under test. For this reason, the selection of the installation point has to take into account the overall behaviour of the structure, the local conditions of the structural parts as well as the actual consistency of the constituent material, including blocks (stones or bricks), mortars and the interaction between them.

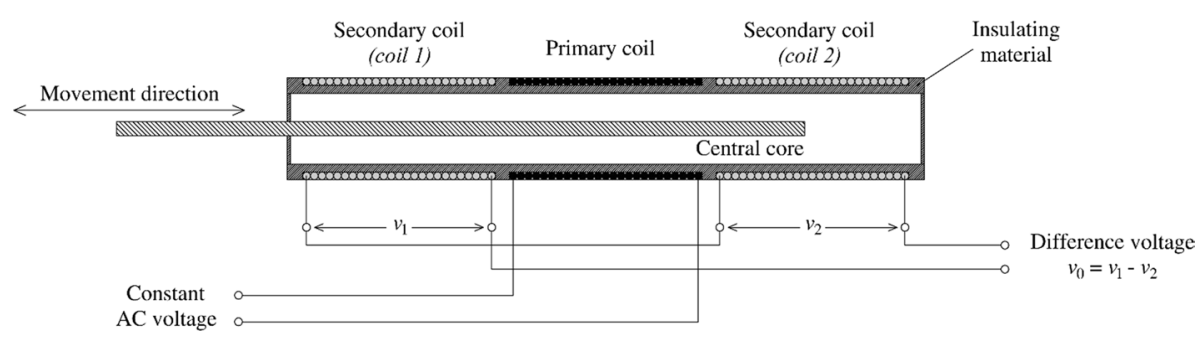

Fig. 1 Scheme illustrating the working principle of a linear variable differential transducer 


\subsection{Ground Penetrating Radar}

GPR working principle is similar to any conventional radar. A transmitting antenna radiates an electromagnetic signal, belonging to the microwave frequency range, into the probed medium and when the radar wave impinges on a target (i.e. an electromagnetic perturbation of the probed medium) part of the energy is backscattered and captured by the receiving antenna. The backscattered signal is measured at a certain time instant after the transmission, and this delay denotes the travel time of the wave along the transmitter-target-receiver path. Therefore, the signal measured at a single position of the antenna system is a time-dependent waveform, referred as A-scan, whose amplitude is not negligible when the electromagnetic wave is reflected by an anomaly. During a GPR survey, the antenna system is moved along a profile, referred as GPR trace, the antenna position is recorded usually by means of an encoder and the waveforms are collected at evenly spaced points along the GPR trace and joined to form a spatial-time image, referred to as raw-data "radargram" or B-scan (Daniels 2004; Persico 2014). Therefore, GPR is an electromagnetic sensing tool exploiting microwaves capability of penetrating into non-metallic media and performing a non-invasive characterization of the scenario under test. Specifically, the result of a GPR survey is an image, which shows the features of the investigated scenario into a certain range of depths, starting from the measurement line/surface up to a depth value, which depends on the signal attenuation into the probed medium and on the choice of observation time window (Daniels 2004; Jol 2009; Persico 2014).

Thanks to these characteristics, in the last three decades, the GPR has received considerable attention in a wide range of applicative scenarios. In fact, the literature is rich with experimental studies assessing the GPR imaging capabilities in on-field scenarios, such as subsoil investigation, infrastructures and cultural heritage diagnostic, archaeologically prospections and so on (Daniels 2004; Jol 2009; Masini and Soldovieri 2017). For instance, in archaeology, GPR allows the detection of areas with alleged interesting buried remains, thus making possible to avoid exhaustive and expansive excavations (Conyers 2013). As a further example, for the monitoring of monuments such as historical buildings, statues (Sambuoelli et al. 2011), ancient fountains, historical bridges (Solla et al. 2011; Bavusi et al. 2010) and so on, GPR gives information useful to assess their conservation and vulnerability state, allowing a characterization of their inner structure and the detection of possible risks, such as the presence of inner voids and/or water infiltrations, and not directly visible anomalies. Moreover, GPR could be useful to check the effectiveness of restoration activity and can be used to achieve information of historical/architectural interest such as about the presence of the walled rooms, hypogeum rooms, tombs and so on (Pieraccini et al. 2006; Grasso et al. 2011). Finally, GPR prospecting is also exploited in civil engineering widely because it permits us to detect and localize structural damages (Catapano et al. 2012; Kadioglu et al. 2013) and hidden structures, like sewers or water and gas pipes, whose presence is in many cases not documented (Hasan and Yazdani 2014; Chang et al. 2009; Capozzoli and Rizzo 2017).

However, the results of a GPR survey, i.e. a raw radargram, are a distorted representation of the scenario under test and its interpretability may strongly depend on the user expertise. In order to improve the interpretability of the radargrams, beyond filtering procedures devoted to remove undesired signals (i.e. direct antenna coupling, air-material reflection, clutter and noise), microwaves tomography approaches are herein used to perform GPR data processing (Leone and Soldovieri 2003; Solimene et al. 2014). In particular, a Born approximation-based microwave tomography approach is herein exploited to obtain 
focused images, from which it is possible to retrieve information about the geometrical parameters (i.e. location, size and shape) of the objects. The adopted data processing strategy is summarized in Fig. 2 and is organized into two main key phases: pre-processing and data inversion. As far as the pre-processing is concerned, it involves several filtering procedures in the time domain, such as the zero-time correction, time gating (TG) and background removal (BKR), which allow us to remove direct antenna coupling and to reduce noise, thus emphasizing the scattered field due to the target. Specifically, the time domain procedure begins with the zero-time correction, which allows us to erase the first part of the B-scan up to the time instant at which the receiving antenna begins to collect the backscattered field. The TG procedure, instead, forces to zero the portion of the radargram corresponding to the antenna direct coupling or, more in general, the radargram portion that is outside the time window where the signal due to the targets of interest is expected. Finally, the BKR consists in replacing the current radar trace, referred to as A-scan, with the difference between it and the average value of all the A-scans as collected along the measurement axis (or a part of it). This operation erases all the flat interfaces present in the data and usually provides a cleaner image of the buried scenario.

Afterwards, the filtered radargram is processed by means of a microwave tomographic approach. This latter faces the imaging as an inverse scattering problem and utilizes the Born approximation to define, into the frequency domain, the mathematical model describing the relationship between the scattered field $E_{\mathrm{s}}\left(\boldsymbol{r}_{\mathrm{m}}, \omega\right)$ data, i.e. the filtered radargram, and the unknowns, i.e. the targets, which are looked for as electromagnetic anomalies with respect to the electromagnetic features of the background medium. Accordingly, the imaging is formulated as the reconstruction of the unknown electric contrast $\chi(\mathbf{r})=\left(\varepsilon_{x}(\mathbf{r}) / \varepsilon_{b}-1\right)$, where $\mathbf{r}$ denotes the generic point belonging to the investigated spatial domain $D, \varepsilon_{x}(\mathbf{r})$ is the unknown permittivity and $\varepsilon_{b}$ the dielectric permittivity of the probed medium. In more detail, for each measurement position $\mathbf{r}_{\mathrm{m}}$ and angular frequency $\omega$, the scattering phenomena are described by means of the linear integral equation:

Fig. 2 Block diagram of the data processing strategy

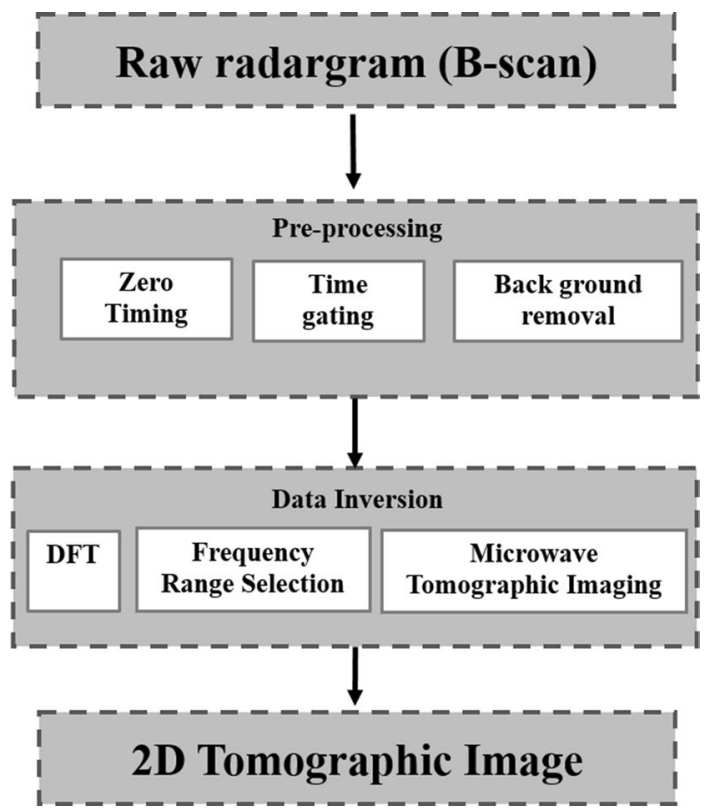




$$
E_{\mathrm{s}}\left(\boldsymbol{r}_{\mathrm{m}}, \omega\right)=k_{\mathrm{b}}^{2} \iint_{D} G\left(\boldsymbol{r}_{\mathrm{m}}, \boldsymbol{r}, \omega\right) E_{\mathrm{inc}}(\boldsymbol{r}, \omega) \chi(\boldsymbol{r}) \mathrm{d} \boldsymbol{r}
$$

In Eq. (1), $k_{\mathrm{b}}$ is the wave number in the background medium, while $E_{\mathrm{inc}}$ and $G$ are the incident field and the Green's function, respectively. Note that $E_{\text {inc }}$ is the field radiated by a line source fed by a unit current. By applying the method of moments to discretize eq. (1), the imaging is faced by solving the matrix inversion problem:

$$
\boldsymbol{E}_{\mathrm{s}}=\boldsymbol{A}[\chi]
$$

where $\boldsymbol{E}_{\mathrm{s}}$ is the $K=M \times F$ dimensional data vector, $M$ being the number of spatial measurement points and $F$ the number of working frequencies, $\chi$ is the $N$ dimensional unknown vector, $N$ being the number of points in $D$ and $\mathbf{A}$ is the $K \times N$ dimensional matrix obtained by discretizing the integral operator in Eq. (1). The matrix inversion problem stated by Eq. (2) is ill-conditioned, and its regularized solution is given by:

$$
\tilde{\chi}(\boldsymbol{r})=\sum_{n=1}^{Q} \frac{\left\langle\boldsymbol{E}_{\mathrm{s}}, \boldsymbol{u}_{n}\right\rangle}{\sigma_{n}} \boldsymbol{v}_{n}(\boldsymbol{r})
$$

In Eq. (3), $\langle\bullet, \bullet\rangle$ denotes the scalar product in the data space, $Q$ is the truncation threshold, $\left\{\sigma_{n}\right\}_{n=1}^{K}$ is the set of singular values of the matrix $\mathbf{A}$ ordered in a decreasing way and $\left\{\boldsymbol{u}_{n}\right\}_{n=1}^{K}$ and $\left\{\boldsymbol{v}_{n}\right\}_{n=1}^{K}$ are the sets of the singular vectors. The threshold $Q \leq K$ defines the "degree of regularization" of the solution, and it is chosen as a trade-off between accuracy and resolution requirement from one side (which should push to increase the $Q$ value) and solution stability from the other side (which should push to limit the value of $Q$ ).

It is worth mentioning that the formulation of the imaging as a linear inverse scattering approach allows a theoretical analysis of the reconstruction performances in terms of the available spatial resolution limits for a fixed measurement configuration. Conversely, it provides guidelines about the measurement configuration to be used, i.e. the spatial offset among the measurement points and the frequency sampling to be adopted in order to move towards the desired imaging performances (Persico 2014; Solimene et al. 2014; Gennarelli et al. 2015; Soldovieri et al. 2007, 2017).

\section{The Case Study}

Gubbio (Italy) is a historical town, located in the Central Italy, whose origin dates back to the pre-Roman time and reached its maximum magnificence during the Middle Ages. The town is characterized by many important and spectacular historical buildings, such as Consoli Palace, Roman theatre, Saint Francesco and Saint Giovanni churches, Medieval Walls, which makes it possible to consider Gubbio as an open-air and living museum.

Consoli Palace is considered the symbol of Gubbio and is the most representative building of the monumental town. It has a rectangular plan, whose size is about $40 \times 20 \mathrm{~m}^{2}$, an elevation of more than $60 \mathrm{~m}$ (from the street level up to the top of the bell tower), and, in terms of building materials, it is mainly made up of calcareous stone masonry. Moreover, the Palace is characterized by an articulated internal distribution of volumes. Indeed, given the slope of the mountain on which it is erected, the Palace foundations sit above two terraces corresponding to the building's lower floors; those 
of the projecting southward (the "Loggia") are placed approximately $10 \mathrm{~m}$ below those of the top, see Fig. 3a. This figure also provides a representation of the crack pattern affecting the main façade of the Consoli Palace (East façade), which are visible form Piazza Grande. The Consoli Palace shows, indeed, evidence of significant structural damages in the form of existing cracks, which were the effects of natural hazards (e.g., earthquakes) and environmental long-term actions that have dragged the Palace over the centuries. The presence of this diffused cracking phenomenon together with the ongoing material degradation motivated the choice of the Consoli Palace as one of the test sites of the HERACLES project (http://www.heracles-project.eu/), and several sensing technologies are currently deployed to investigate and monitor its structural conditions.

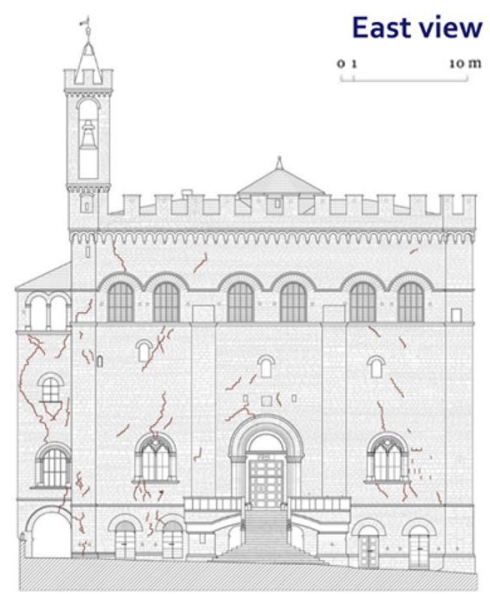

(a)

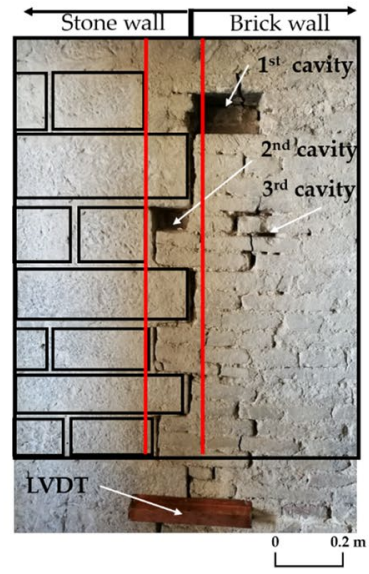

(c)

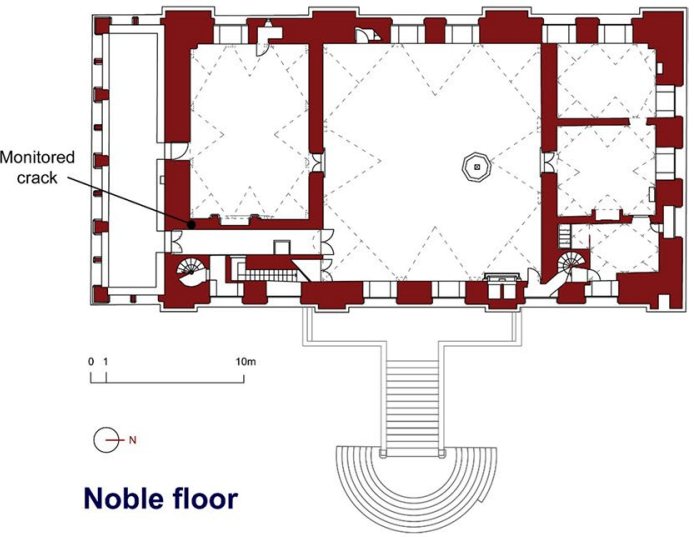

(b)

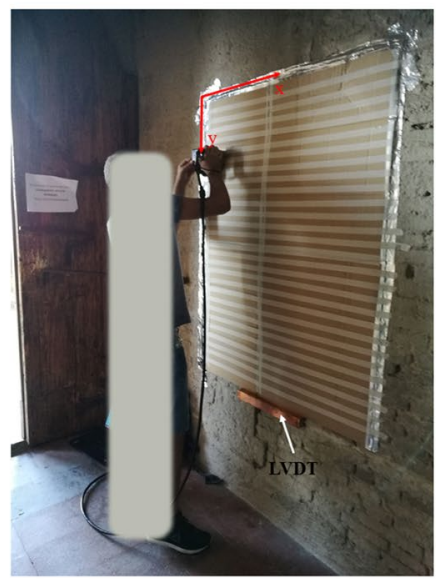

(d)

Fig. 3 a East view of Consoli Palace showing the external crack patterns; $\mathbf{b}$ plan of the Noble floor of the Consoli Palace, with indication of the monitored crack; c picture of the crack pattern and the three main cavities on the wall along the Loggia cross-hall. The red lines delimit the junction area between stone and brick walls, d GPR investigated area covered by a cardboard and having side $0.90 \mathrm{~m}$ along the $x$-axis and $1.1 \mathrm{~m}$ along $y$-axis 
Among the structural damages affecting the Palace, on the indications of the stakeholder managing it, we have paid attention on a widespread crack pattern well visible in the crosshall of the Loggia, at the level of the so called "Noble floor" (see Fig. 3b). Figure $3 c$ shows that the crack is located between two different walls, a stone wall (left), which is part of the external perimeter of the Palace, and a brick wall (right), which is an internal wall separating the cross-hall from a large hall. Specifically, the crack pattern occurs at the junction area of these two walls. Figure $3 \mathrm{c}$ also shows the presence of three main cavities, due to the fall of some bricks due to the crack, and the arrangement of stones and bricks. In particular, it is possible to observe that the left part is made up by alternating stone blocks whose lengths are $0.40 \mathrm{~m}$ and $0.70 \mathrm{~m}$, respectively.

In order to assess and monitor this crack pattern, a LVDT has been installed and activated since July 2017 and two GPR measurement surveys were performed in 12 July 2017 and 8 May 2018, respectively. Figure 3c, d shows the LVDT sensor, covered by a protection copper box, whereas the rectangular area outlined by the black contour in Fig. 3c denotes the spatial region investigated by GPR surveys. This latter appears covered by a cardboard in Fig. $3 \mathrm{~d}$, and it is $0.90 \mathrm{~m}$ along the $x$-axis and $1.1 \mathrm{~m}$ along $y$-axis.

\section{Results of the Survey Activities}

\subsection{One-Year LVDT Monitoring of the South Façade}

The South façade of the palace, comprising also the structure of the Loggia, is characterized by a complex damage scenario, involving the detachment of the Loggia from the façade and the façade itself. The LVDT, Solartron Metrology S-model with a linear measurement range from 0 to $50 \mathrm{~mm}$ and a resolution $<0.3 \mu \mathrm{m}$, was installed together with a K-type thermocouple (T1) across the crack investigated in this work (see Fig. 4). In particular, the crack belongs to a complex damage scenario, which is ascribed to several actions: horizontal shear forces acting in the plane of the South façade, detachment of internal walls from the façade and possible out-of-plane movements of the Loggia.

The monitoring period started on 5 July 2017, and the data have been recorded through a data acquisition system, model NI CompactDAQ-9132, supplied through an uninterruptible power supply (UPS), with the following technical characteristics: processor $1.33 \mathrm{GHz}$ dual-core atom, 2 GB RAM, 16 GB SD storage, 4-Slot, Windows Embedded Standard

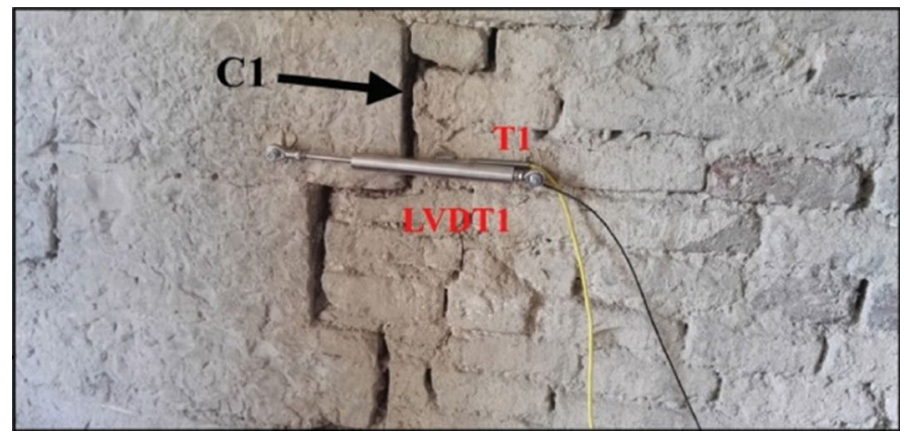

Fig. 4 Images of the static monitoring system: LVDT1 and thermal sensor T1 installed in the South façade 


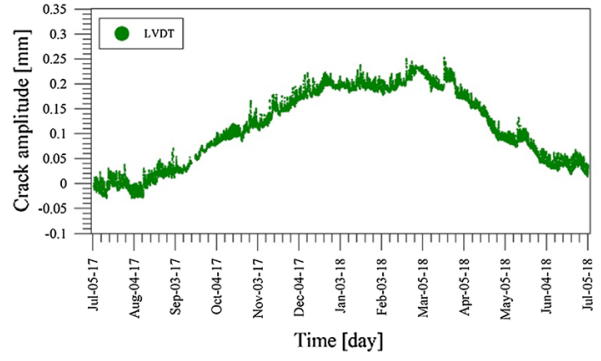

(a)

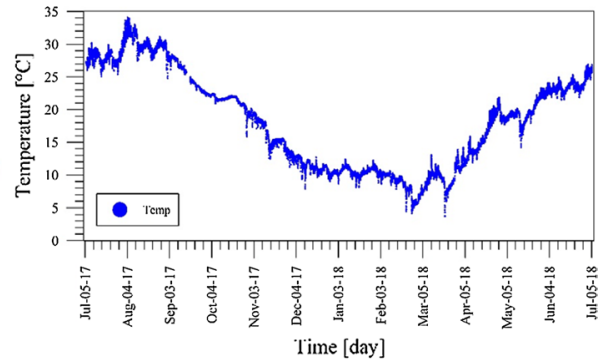

(b)

Fig. 5 Displacement (a) and temperature (b) time histories recorded by the LVDT and T1 sensors, respectively

Fig. 6 RIS K2_FW GPR architecture system

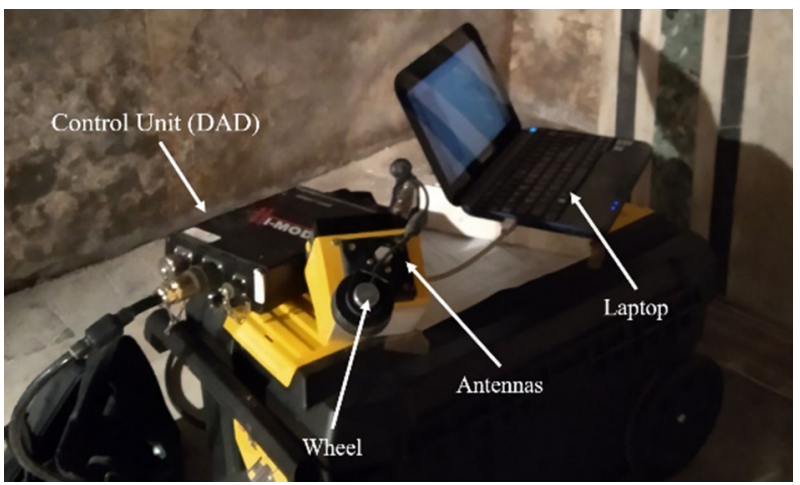

7 operating system. The data have been stored in consecutive separate files containing 30 min recordings and sent through the Internet to the remote server located in the Laboratory of Structural Dynamics of University of Perugia for the post-processing.

Figure 5a shows the displacement time history recorded by the LVDT sensor, where the increasing trend of measurements indicates an opening of the crack. It should be noted that in the first year of monitoring the observed range of displacement is of about $0.3 \mathrm{~mm}$, which can be considered as a relatively small amplitude change. Figure $5 \mathrm{~b}$ shows the temperature time history recorded by the $\mathrm{T} 1$ sensor in the same period; specifically, the temperature variation covers a range from about $5{ }^{\circ} \mathrm{C}$ to $35^{\circ} \mathrm{C}$ and has a trend that is coherent with the seasonal changes.

\subsection{GPR Investigations}

The measurement surveys at the Consoli Palace were performed by using the IDS-manufactured RIS K2_FW GPR system (https://idsgeoradar.com/products) equipped with a 2-GHz single-fold shielded antennas, see Fig. 6.

The RIS K2_FW is a time domain system, whose antennas, transmitting and receiving, are incorporated in a rigid structure and moved together. In addition, for the measurement procedure, the library TRHF model was considered, which corresponds to the adopted antenna system and uses a 32-ns time window discretized by 512 samples. Each radargram, 
or B-scan, is gathered by moving the system by hand along the measurement profile, i.e. a straight line. A survey wheel synchronized spatial movement and data acquisition.

In order to facilitate the antenna movement, a cardboard was applied on the investigated region during the measurement step, which covered a surface of about $1 \mathrm{~m}^{2}$, whose side is $0.90 \mathrm{~m}$ along the $x$-axis and $1.1 \mathrm{~m}$ along $y$-axis, according to the coordinate reference system depicted in Fig. 3d. Data were gathered with a $0.01-\mathrm{m}$-spatial offset by moving the antenna from left to right along $x$-axis and by considering 25 traces, $0.05 \mathrm{~m}$ spaced one to each other, along the $y$-axis.

The raw data, collected during both the GPR campaigns, were processed by using the same data processing chain described in previous Section. The output is an image showing the 3D spatial distribution of the amplitude of the reconstructed electric contrast as normalized to its maximum value, and it is herein represented by means of constant depth slices, which for brevity are referred to as tomographic images (Catapano et al. 2017).

Figure $7 \mathrm{a}-\mathrm{j}$ shows a qualitative comparison between the constant depth slices retrieved by the tomographic images obtained during the two measurement campaigns. In particular, Fig. 7a-f shows the tomographic images at depths $z=0.03 \mathrm{~m}, z=0.07 \mathrm{~m}$ and $z=0.12 \mathrm{~m}$, respectively, which are referred to as the shallower part of the investigated spatial domain. Conversely, Fig. $7 \mathrm{~g}-\mathrm{j}$, which shows the constant depth slices at $z=0.50 \mathrm{~m}$ and $z=0.70 \mathrm{~m}$ respectively, is concerned with the middle and bottom part of the investigated volume.

Similar to the results of the first GPR campaign (Catapano et al. 2017), the tomographic images referred to the second survey and corresponding to $z=0.03 \mathrm{~m}$ show the crack pattern, three main cavities and the arrangement of the stone blocks (see Fig. 7a, b). These elements, as said, are clearly recognizable by means of a visual inspection (see Fig. 3c). Whereas, as an added value, the tomographic images allow us to infer the depth of the cavities, the arrangement of the stone blocks in the inner part of the wall and the end of the brick wall. Specifically, based on Fig. 7c, d, wherein the third cavity is not well visible, one can infer that this cavity is less deep compared to the other two cavities and the crack pattern. Figure 7e, f shows that only the first cavity is still present and one can infer that the crack reaches a depth smaller than about $0.10 \mathrm{~m}$. In addition, Fig. $7 \mathrm{~g}, \mathrm{~h}$ shows three long stone blocks, which maybe were adopted for the junction of the walls, while Fig. 7i, j shows the interface between stone and brick walls, which has been marked by the vertical white line. Moreover, in Fig. 7i, j, the texture of the brick wall is recognizable due to the occurrence, at this depth, of the backside of the wall, i.e. the brick wall-air interface that arises an electromagnetic reflection collected by GPR. The thickness of the brick wall is indeed about $75 \mathrm{~cm}$.

Furthermore, in order to emphasize the advantages offered by the microwave tomographic approach, Fig. 8a-j shows the constant depth slices, obtained by processing the data collected during the two GPR surveys only by applying the pre-processing step (zero time, time gating and background removal). These slices are referred to the same $z$ (depth) values of the ones considered for the tomographic images. As far as the shallower part of the investigated spatial domain is concerned, the reconstructions shown in Fig. 8a-f, with respect to the tomographic images in Fig. 8a-f, do not clearly highlight the presence of the three main cavities and the arrangement of the stone blocks. Moreover, in the deeper investigated parts, the three long stone blocks and the interface between stone and brick walls, as well as their texture, are not recognizable (see Fig. $8 \mathrm{~g}-\mathrm{j}$ ). 
Fig. 7 A qualitative comparison at constant depth slices between the tomographic images retrieved by the data acquired on 12 July 2017 (a, c, e, g, i panels) and 8 May 2018 (b, d, f, h, j panels). The tomographic images show the normalized intensity of the retrieved contrast function
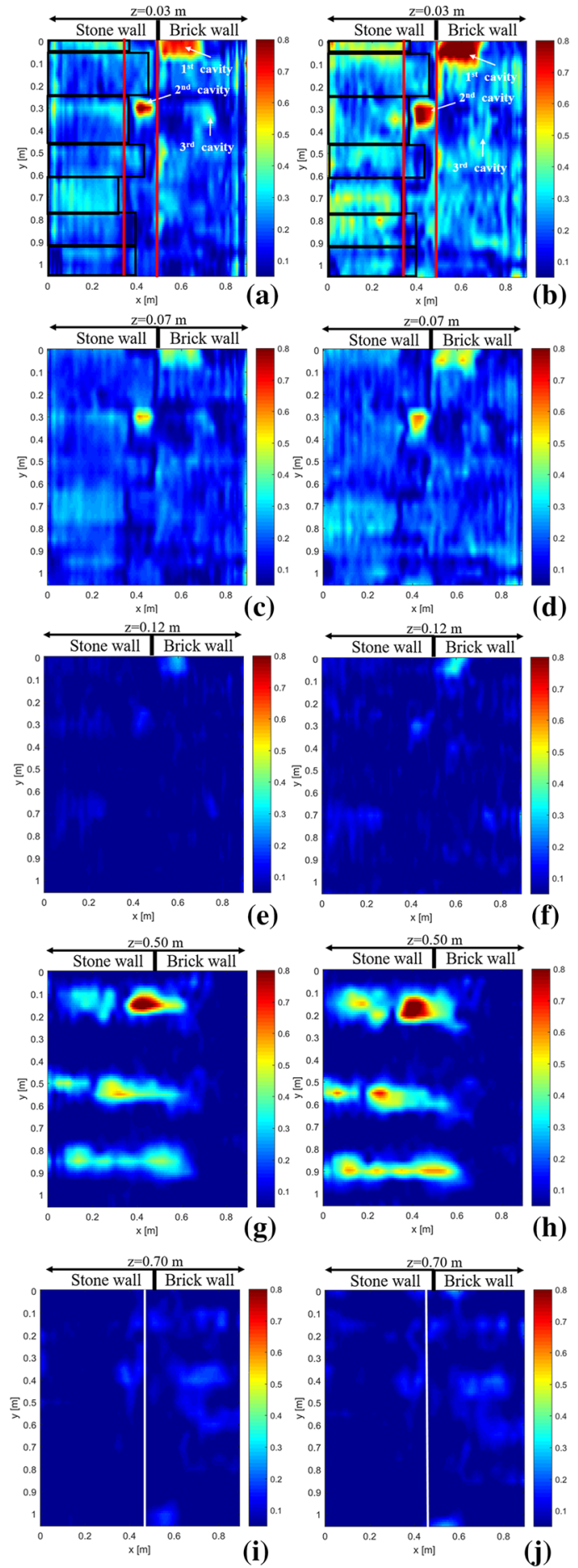
Fig. 8 Qualitative comparison at constant depth slices between filtered radargram referred to data measured on 12 July 2017 (a, c, e, g, i panels) and 8 May 2018 (b, $\mathbf{d}, \mathbf{f}, \mathbf{h}, \mathbf{j}$ panels). The images show the normalized intensity of the filtered radargrams
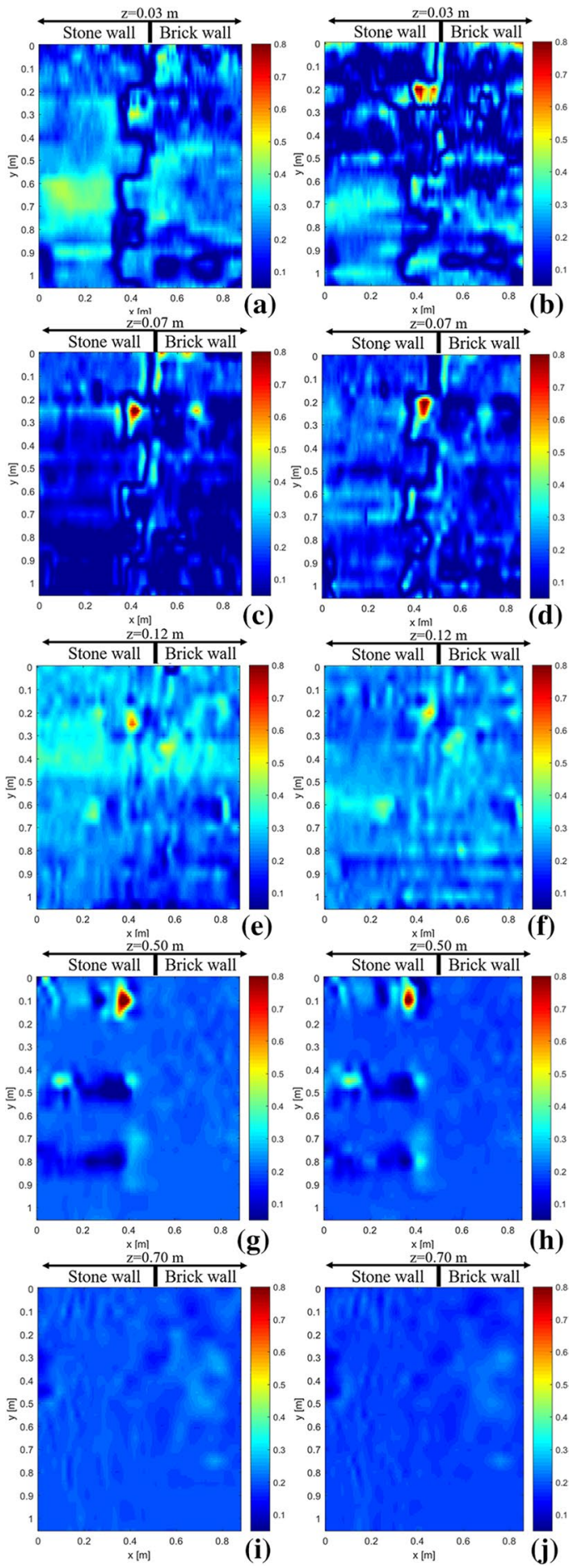


\section{Discussion About the Combined Use of the Sensing Techniques}

The crack amplitude time history recorded by the LVDT1 sensor and the two GPR surveys performed on the crack of the Loggia cross-hall allowed us to obtain information related to the time behaviour of the cracking phenomenon. Specifically, as shown in Fig. 9a, the maximum displacement was recorded by the LVDT sensor at the end of the winter season (see Fig. 9b) and is about equal to $0.3 \mathrm{~mm}$. Moreover, this value corresponds to the minimum temperature value recorded by the $\mathrm{T} 1$ sensor, as shown by the visual correlation between displacement and temperature data in Fig. 9a. In more detail, an opening of the crack has been observed with a decrease of the temperature. This behaviour is due to the material contraction correlated with the low level of temperature, which caused the opening of the crack. Nevertheless, it can be observed that the fluctuation amplitude is smaller than $1 \mathrm{~mm}$, highlighting no significant change of crack amplitude due to structural hazards. Moreover, Fig. 9b shows the estimation of the linear regression parameters, which allow us to infer that the evolution of the crack amplitude is related to the seasonal temperature change.

As far as GPR tomographic images are concerned, the qualitative comparison at the increasing depths, shown in Fig. 7a-e, did not highlight significant differences between the two measurement campaigns. In fact, as for the result presented in Catapano et al. (2017), also the tomographic reconstructions obtained from the data of the second survey allow us to infer that the crack affecting this zone reaches a depth $z$ of about $0.10 \mathrm{~m}$, while the first, second and third cavities reach depths about $0.13 \mathrm{~m}, 0.11 \mathrm{~m}$ and $0.07 \mathrm{~m}$, respectively. In addition, also from the depth slice $z=0.50 \mathrm{~m}$, it is still possible to retrieve information about the three stone blocks, which are $\mathrm{z}=0.70 \mathrm{~m}$ long and whose arrangement is similar to that of the surface stones (see Fig. 3c).

In order to corroborate, in a quantitative way, these statements, i.e. that 1 year later the wall structure, did not change, a statistical analysis has been performed. Specifically, we used for this analysis the correlation coefficient $(R)$ and the root mean square error (RMSE) between two tomographic images referred to the same depth value. $R$ and RMSE were computed by means Eqs. (4) and (5), respectively.

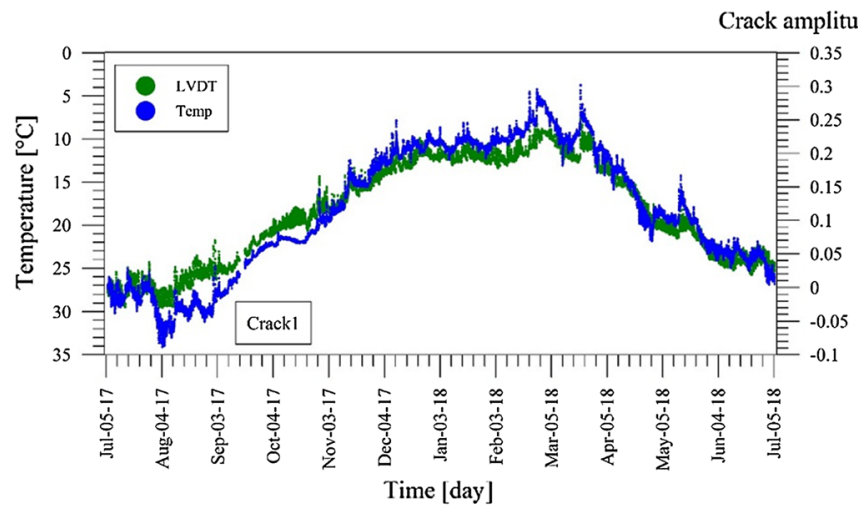

(a)

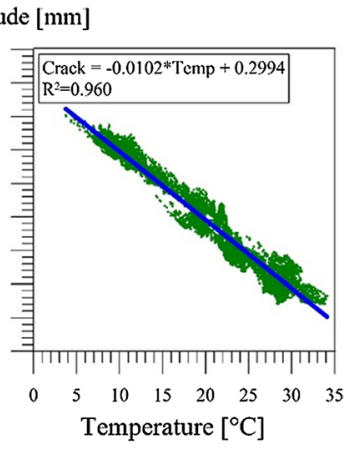

(b)

Fig. 9 Correlation between the displacement and temperature data acquired in the crack of the South façade 


$$
\begin{gathered}
R=\frac{\sum_{l=1}^{L} \sum_{p=1}^{P}\left(I 1_{l, p}-\overline{I 1}\right)\left(I 2_{l, p}-\overline{I 2}\right)}{\sqrt{\left(\sum_{l=1}^{L} \sum_{p=1}^{P}\left(I 1_{l, p}-\overline{I 1}\right)^{2}\right)\left(\sum_{l=1}^{L} \sum_{p=1}^{P}\left(I 2_{l, p}-\overline{I 2}\right)^{2}\right)}} \\
\operatorname{RMSE}=\sqrt{\frac{1}{\mathrm{LP}} \sum_{l=1}^{L} \sum_{p=1}^{P}\left|\Delta_{l, p}\right|^{2}}
\end{gathered}
$$

In Eq. (4), $I 1$ and $I 2$ represent the tomographic images of the first and second measurement campaign, respectively, while $\bar{I}$ and $\bar{I} 2$ are the corresponding average values. In Eq. (5), instead, $\Delta=(I 1-I 2)$ represents the image difference between two tomographic images and referred to the same depth value. In addition, for both equations, $l=1, \ldots, L$ and $p=1, \ldots, P$ represent the indices along $x$-axis and $y$-axis of each depth slice, respectively.

Figure 10 shows the behaviour along the $z$-axis of the $R$ and the RMSE between two tomographic images, one for each GPR survey, and referred to the same depth value. The behaviour of the correlation coefficient is characterized by an average $R$ value of about $60 \%$ for depths from $0 \mathrm{~m}$ up to $0.4 \mathrm{~m}$ and of about $80 \%$ for the remaining depths. On the other hand, the RMSE has an average value of about $15 \%$ for all considered depths. The low $R$ value for the first part of the wall depth is not attributable to the evolution of the crack during the time between two surveys, but rather to the different climate conditions at the time of the two GPR acquisitions and to the fact that these latter were performed by two different persons. In this frame, it is worth pointing out that the LVDT sensor has recorded a displacement at most of $0.3 \mathrm{~mm}$, which is significantly lower than the cross-range resolution provided by the GPR system. Indeed, by taking into account that the nominal bandwidth and the nominal central frequency are both equal to $2 \mathrm{GHz}$, the range resolution of the GPR system is about $4 \mathrm{~cm}$, while the cross-range resolution is about $7.5 \mathrm{~cm}$.

On the other hand, by accounting for the two different temperature values measured during the first and second surveys, i.e. $25{ }^{\circ} \mathrm{C}$ and $20{ }^{\circ} \mathrm{C}$ (see Fig. $5 \mathrm{~b}$ ), it is foreseeable that a

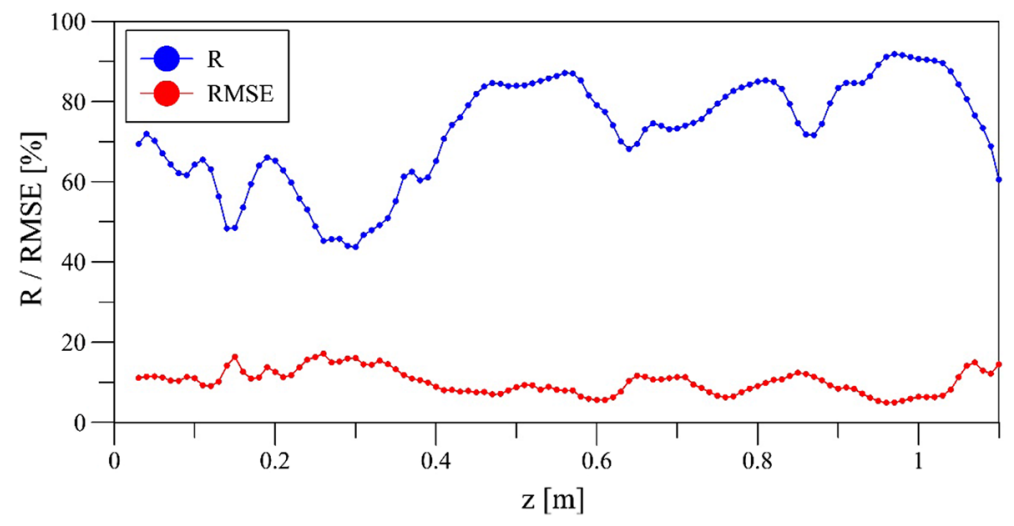

Fig. 10 Behaviour of the correlation coefficient $(R)$ (blue line) and root mean square error (RMSE) (orange line) between the tomographic images at same depth retrieved from the two surveys 
small change of the electromagnetic characteristic of the investigated materials occurred. Such a change mainly involved materials at the shallow part of the domain under test, due to the changes of their humidity content. Consequently, the correlation between the tomographic reconstructions referred to the two surveys show a lower correlation at the first depth values than elsewhere.

According to LVDT and GPR results obtained in 1 year of monitoring, it is possible to highlight that no significant evolution of the crack was evidenced and only changes related to the thermal behaviour of the materials were experienced. Considering that the Consoli Palace is affected by a moderate damage state, the monitoring of the time evolution of its actual damage state needs to be continued to understand its stability.

\section{Conclusions}

In the general frame of $\mathrm{CH}$ structural monitoring, the present paper has presented an illustrative case of joint use of the LVDT and GPR for the diagnosis and monitoring of a crack located in the cross-hall of the Loggia at Consoli Palace in Gubbio, Italy. LVDT recorded data related to the amplitude of the crack during 1 year of observation, starting from July 2017, and the main outcome was that the opening behaviour of the crack followed a concave parabolic trend in time, with maximum value equal to $0.3 \mathrm{~mm}$. In addition, the correlation of the displacement trend with temperature allowed us to infer that the observed crack amplitude changes were due to the seasonal climate changes that caused a material contraction, which reached its maximum value with the lowest seasonal temperature value.

As far as the GPR survey is concerned, the tomographic reconstructions retrieved from the two surveys performed on 12 July 2017 and 8 May 2018 allowed the characterization of the surface and the interior of the wall. These results have provided information about the depth of the crack and of the three main cavities, as well as about the arrangement of the stone blocks and the texture of the bricks. Moreover, a statistical analysis, in terms of correlation coefficient $(R)$ and root mean square error (RMSE), has been performed to compare the results of the two surveys. The analysis has shown small variations that can conceivably be attributed to the two different temperature values measured during the first and second surveys. It is, indeed, foreseeable that a small change of the electromagnetic characteristic of the investigated materials, mainly those at the shallow part of the domain under test, occurred due to the changes of their humidity content.

Finally, both the low value of the maximum opening of the crack recorded by the LVDT sensor and the high $R$ value and low RMSE value obtained from the comparison of the results of the two surveys allowed us to conclude that no significant structural damages occurred in the investigated area during the observation time.

On-going activities at Consoli Palace, in the frame of HERACELS project, are addressing the joint use and the correlation of the presented results with those provided by other sensing technologies, among which synthetic-aperture radar interferometry performed by satellite and the assimilation of the results of the integrated sensing approach into a structural finite element method (FEM) model. The main aim of FEM model is to investigate the effects of differential settlements of foundations-probably due to soil movements over decades and/or climate-change related effects (e.g., rain 
falls and drought periods) - and exceptional strong events, such as earthquakes, on the stability of the structure. In this frame, diffused crack scenarios observed in the façades and the indoor of the building could be related to a combined action of both the stress levels developed inside the material and the degradation of the mortars in the joints between the stones, mainly due to environmental actions.

Acknowledgements This project has received funding from the European Union's Horizon 2020 research and innovation programme under Grant Agreement No. 700395.

Open Access This article is distributed under the terms of the Creative Commons Attribution 4.0 International License (http://creativecommons.org/licenses/by/4.0/), which permits unrestricted use, distribution, and reproduction in any medium, provided you give appropriate credit to the original author(s) and the source, provide a link to the Creative Commons license, and indicate if changes were made.

\section{References}

ASCE (2013) Report Card for America's Infrastructure. www.infrastructurereportcard.org. Accessed 28 Jan 2019

Baeza FJ, Ivorra S, Bru D, Varona FB (2018) Structural health monitoring systems for smart heritage and infrastructures in Spain. In: Ottaviano E, Pelliccio A, Gattulli V (eds) Mechatronics for cultural heritage and civil engineering, vol 92. Intelligent Systems, Control and Automation: Science and Engineering. Springer, Cham

Bavusi M, Soldovieri F, Piscitelli S, Loperte A, Vallianatos F, Soupios P (2010) Ground penetrating radar and microwave tomography to evaluate the crack and joint geometry in historical buildings: some examples from Chania, Crete, Greece. Near Surf Geophys 8(5):377-387

Blanco H, Boffill Y, Lombillo I, Villegas L (2018) An integrated structural health monitoring system for determining local/global responses of historic masonry buildings. Struct Control Health Monit 25(8):e2196

Bolton W (2015) Chapter 2-Instrumentation system elements. In: Instrumentation and control systems, 2nd edn. Newnes, pp 15-65

Burrows SE, Rashed A, Almond DP, Dixon S (2007) Combined laser spot imaging thermography and ultrasonic measurements for crack detection. Nondestruct Test Eval 22(2):217-227

Cabboi A, Gentile C, Saisi A (2017) From continuous vibration monitoring to FEM-based damage assessment: Application on a stone-masonry tower. Constr Build Mater 156:252-265

Capozzoli L, Rizzo E (2017) Combined NDT techniques in civil engineering applications: laboratory and real tests. Constr Build Mater 154:1139-1150

Catapano I, Di Napoli R, Soldovieri F, Bavusi M, Loperte A, Dumoulin J (2012) Structural monitoring via microwave tomography-enhanced GPR: the Montagnole test site. J Geophys Eng 9:S100-S107

Catapano I, Ludeno G, Soldovieri F, Tosti F, Padeletti G (2017) Structural assessment via ground penetrating radar at the Consoli Palace of Gubbio (Italy). Remote Sens 10:45

Ceravolo R, De Marinis A, Pecorelli ML, Zanotti Fragonara L (2017) Monitoring of masonry historical constructions: 10 years of static monitoring of the world's largest oval dome. Struct Control Health Monit 24(10):e1988

Chang CW, Lin CH, Lien HS (2009) Measurement radius of reinforcing steel bar in concrete using digital image GPR. Constr Build Mater 23:1057-1063

Chen J, Bulbul T, Taylor J, Olgun G (2014) A case study of embedding real-time infrastructure sensor data to BIM. In: Construction research congress, pp 269-278

Chen Z, Zhou X, Wang X, Dong L, Qian Y (2017) Deployment of a smart structural health monitoring system for long-span arch bridges: a review and a case study. Sensors 17:2151

Chong KP, Carino NJ, Washer GA (2001) Health monitoring of civil infrastructures. In: Proceedings on SPIE 4337, health monitoring and management of civil infrastructure systems (3 August 2001). https://doi.org/10.1117/12.435595

Cigada A, Corradi Dell'Acqua L, Mörlin Visconti Castiglione B, Scaccabarozzi M, Vanali M, Zappa E (2017) Structural health monitoring of an historical building: the main spire of the Duomo Di Milano. Int J Archit Herit 11(4):501-518

Coïsson E, Blasi C (2015) Monitoring the French Panthéon: from Rondelet's historical surveys to the modern automatic system. Int J Archit Heri Conserv Anal Restor 9(1):48-57 
Coïsson E, Ottoni F (2015) Structural monitoring of historical constructions: increasing knowledge to minimize interventions. In: Toniolo L, Boriani M, Guidi G (eds) Built heritage: monitoring conservation management. Research for development. Springer, Cham, pp 83-92

Conyers LB (2013) Ground penetrating radar for archaeology, 3rd edn. Altamira Press, Lanham

Daniels DJ (2004) Ground penetrating radar, vol 15. IEE Radar, Sonar and Navigation Series. IEE, London

Deutscher Bundestag (2014) German Bundestag, Committee on transport and digital infrastructure, Berlin, Germany. www.bundestag.de/dokumente/textarchiv/2014/49449197_kw07_pa_verkehr/21564 4. Accessed 28 Jan 2019

Diamanti N, Annan AP, David Redman J (2017) Concrete bridge deck deterioration assessment using Ground Penetrating Radar (GPR). JEEG 22(2):121-132

Duvnjak I, Damjanović D, Krolo J (2016) Structural health monitoring of cultural heritage structures: applications on peristyle of Diocletian's palace in split. In: 8th European workshop on structural health monitoring, EWSHM 2016, vol 4, pp 2661-2669

Eastman C, Teicholz P, Sacks R, Liston K (2008) BIM handbook: a guide to building information modeling for owners, managers, designers, engineers and contractors. Wiley, Hoboken

Frangopol Dan M, Saydam Duygu, Kim Sunyong (2012) Maintenance, management, life-cycle design and performance of structures and infrastructures: a brief review. Struct Infrastruct Eng Maint Manag Life-Cycle Des Perform 8(1):1-25

Gennarelli G, Catapano I, Soldovieri F, Persico R (2015) On the achievable imaging performance in full 3-D linear inverse scattering. IEEE Trans Antennas Propag 63(3):1150-1155

Gioffré M, Gusella V, Cluni F (2008) Performance evaluation of monumental bridges: testing and monitoring 'Ponte delle Torri' in Spoleto. Struct Infrastruct Eng 4(2):95-106

Goodman D, Piro S (2013) GPR remote sensing in archaeology, geotechnologies and the environment, vol 9. Springer, New York

Grasso F, Leucci G, Masini N, Persico R (2011) GPR prospecting in renaissance and baroque monuments in Lecce (Southern Italy). In: Proceedings of VI international workshop on ground penetrating radar, IWAGPR, Aachen, Germany

Hasan I, Yazdani N (2014) Ground penetrating radar utilization in exploring inadequate concrete covers in a new bridge deck. Case Stud Constr Mater 1:104-114

Hejll A, Täljsten B, Carolin A (2006) Structural health monitoring of the Gröndals bridge in Swedenthe behaviour of CFRP strengthening in cold temperature. Proc SPIE Int Soc Opt Eng. https://doi. org/10.1117/12.663987

Jol HM (2009) Ground penetrating radar theory and applications. Elsevier, Amsterdam

Joshi S, Harle SM (2017) Linear Variable Differential Transducer (LVDT) \& its applications in civil engineering. Int J Transp Eng Technol 3(4):62-66

Kadioglu S, Kadioglu YK, Catapano I, Soldovieri F (2013) Ground penetrating radar and microwave tomography for the safety management of a cultural heritage site: Miletos Ilyas Bey Mosque (Turkey). J Geophys Eng 10(6):064007

Kilic G (2015) Using advanced NDT for historic buildings: towards an integrated multidisciplinary health assessment strategy. J Cult Herit 16(4):526-535

Kita A, Cavalagli N, Ubertini F (2019) Temperature effects on static and dynamic behavior of Consoli Palace in Gubbio, Italy. Mech Syst Signal Process 120:180-202

Knudson DL, Rempe JL (2012) Linear Variable Differential Transformer (LVDT)-based elongation measurements in advanced test reactor high temperature irradiation testing. Meas Sci Technol 23(2):025604

Krysiński L, Hugenschmidt J (2015) Effective GPR inspection procedures for construction materials and structures. In: Benedetto A, Pajewski L (eds) Civil engineering applications of ground penetrating radar. Springer Transactions in Civil and Environmental Engineering. Springer, Cham

Leone G, Soldovieri F (2003) Analysis of the distorted born approximation for subsurface reconstruction: truncation and uncertainties effect. IEEE Trans Geosci Remote Sens 41(1):66-74

Leucci G, Masini N, Persico R, Soldovieri F (2011) GPR and sonic tomography for structural restoration: the case of the Cathedral of Tricarico. J Geophys Eng 8:S76-S92

Lombillo I, Blanco H, Pereda J, Villegas L, Carrasco C, Balbás J (2016) Structural health monitoring of a damaged church: design of an integrated platform of electronic instrumentation, data acquisition and client/server software. Struct Control Health Monit 23(1):69-81

Lorenzoni F, Casarin F, Modena C, Caldon M, Islami K, da Porto F (2013) Structural health monitoring of the Roman Arena of Verona, Italy. J Civ Struct Health Monit 3(4):227-246 
Lorenzoni F, Casarin F, Caldon M, Islami K, Modena C (2016) Uncertainty quantification in structural health monitoring: Applications on cultural heritage buildings. Mech Syst Signal Process 66(67):268-281

Mandal H, Bera SK, Saha S, Sadhu PK, Bera SC (2018) Study of a modified LVDT type displacement transducer with unlimited range. IEEE Sens J 18(23):9501-9514

Marazzi F, Tagliabue P, Corbani FM (2011) Traditional vs innovative structural health monitoring of monumental structures: a case study. Struct Control Health Monit 18(4):430-449

Masciotta M-G, Roque JCA, Ramos LF, Lourenço PB (2016) A multidisciplinary approach to assess the health state of heritage structures: the case study of the Church of Monastery of Jerónimos in Lisbon. Constr Build Mater 116:169-187

Masciotta MG, Ramos LF, Lourenço PB (2017) The importance of structural monitoring as a diagnosis and control tool in the restoration process of heritage structures: a case study in Portugal. J Cult Herit 27:36-47

Masini N, Soldovieri F (2011) Special Issue on "Integrated non-invasive sensing techniques and geophysical methods for the study and conservation of architectural, archaeological and artistic heritage". J Geophys, Eng., p 8

Masini N, Soldovieri F (2017) Cultural Heritage sites and sustainable management strategies. In: Masini N, Solodovieri F (eds) Sensing the past. From artifact to historical site. Springer, Basel

Masini N, Persico R, Rizzo E (2010) Some examples of GPR prospecting for monitoring of the monumental heritage. J Geophys Eng 7:190-199

Masini N, Soldovieri F, de Buergo MA, Dumoulin J (2012) Special issue on "Cultural Heritage and Civil Engineering”. J Geophys, Eng., p 4

Masini N, Sileo M, Leucci G, Solodovieri F, D'Antonio A, de Giorgi L, Pecci A, Scavone A (2017) Integrated in situ investigations for the restoration: the case of Regio VIII in Pompei. In: Masini N, Solodovieri F (eds) Sensing the past. From artifact to historical sit. Springer, Basel

Mondal B, Kumar B, Mandal N (2018) Design of an inductive pickup type displacement transducer using an electro-optic effect of lithium-niobate-based Mach-Zehnder interferometers. IET Sci Meas Technol 12(3):395-404

Morris AS, Langari R (2012) Translational motion, vibration, and shock measurement. In: Morris AS, Langari R (eds) Measurement and instrumentation. Butterworth-Heinemann, Oxford, pp 497-528

Noel AB, Abdaoui A, Elfouly T, Ahmed MH, Badawy A, Shehata MS (2017) Structural health monitoring using wireless sensor networks: a comprehensive survey. IEEE Commun Surv Tutor 19(3):1403-1423, art. no. 7894175

Oliveira G, Magalhães F, Cunha Á, Caetano E (2018) Continuous dynamic monitoring of an onshore wind turbine. Eng Struct 164:22-39

Orban Z, Gutermann M (2009) Assessment of masonry arch railway bridges using non-destructive in situ testing methods. Eng Struct 31(10):2287-2298

Ottoni F, Blasi C (2015) Results of a 60-year monitoring system for Santa Maria del Fiore Dome in Florence. Int J Archit Herit 9(1):7-24

Pei C-W (2015) Precision LVDT signal conditioning using direct RMS to DC conversion. In: Dobkin B, Hamburger J (eds) Analog circuit design. Newnes, Oxford, pp 1049-1050

Pérez JPC, de Sanjosé Blasco JJ, Atkinson ADJ, del Río Pérez LM (2018) Assessment of the structural integrity of the Roman Bridge of Alcántara (Spain) using TLS and GPR. Remote Sens 10:387

Persico R (2014) Introduction to ground penetrating radar: inverse scattering and data processing. Wiley, London

Pieraccini M, Noferii L, Mecatti D, Atzeni C, Persico R, Soldovieri F (2006) Advanced processing techniques for step-frequency continuous-wave penetrating radar. the case study of "Palazzo Vecchio" walls (Firenze, Italy). Res Nondestr Eval 17:71-83

Pierdicca A, Clementi F, Fortunati A, Lenci S (2018) Tracking modal parameters evolution of a school building during retrofitting works. Bull Earthq Eng. https://doi.org/10.1007/s10518-018-0483-9 (in press)

Proto M, Bavusi M, Bernini R, Bigagli L, Bost M, Bourquin F, Cottineau LM, Cuomo V, Della Vecchia P, Dolce M, Dumoulin J, Eppelbaum L, Fornaro G, Gustafsson M, Hugenschmidt J, Kaspersen P, Kim H, Lapenna V, Leggio M, Loperte A, Mazzetti P, Moroni C, Nativi S, Nordebo S, Pacini F, Palombo A, Pascucci S, Perrone A, Pignatti S, Ponzo FC, Rizzo E, Soldovieri F, Taillade F (2010) Transport infrastructure surveillance and monitoring by electromagnetic sensing: the ISTIMES project. Sensors 10(12):10620-10639

Rio J, Ferreira B, Pocas-Martins J (2013) Expansion of IFC model with structural sensors. Informes de la Construcciòn 65(530):219-228 
Roselli I, Malena M, Mongelli M, Cavalagli N, Gioffrè M, De Canio G, de Felice G (2018) Health assessment and ambient vibration testing of the "Ponte delle Torri" of Spoleto during the 20162017 Central Italy seismic sequence. J Civ Struct Health Monit 8(2):199-216

Rossi PP, Rossi C (2015) Monitoring of two great Venetian Cathedrals: San Marco and Santa Maria Gloriosa Dei Frari. Int J Archit Herit 9(1):58-81

Russo S (2013) On the monitoring of historic Anime Sante church damaged by earthquake in L'Aquila. Struct Control Health Monit 20(9):1226-1239

Saisi A, Gentile C, Ruccolo A (2018) Continuous monitoring of a challenging heritage tower in Monza, Italy. J Civ Struct Health Monit 8(1):77-90

Sambuoelli L, Bhom G, Capizzi P, Cardarelli E, Cosentino P (2011) Comparison between GPR measurements and ultrasonic tomography with different inversion algorithms: an application to the base of an ancient Egyptian sculpture. J Geophys Eng 8(3):106-116

Sánchez Abraham R, Meli Roberto, Chávez Marcos M (2016) Structural Monitoring of the Mexico City Cathedral (1990-2014). Int J Archit Herit 10(2-3):254-268

Sankarasrinivasan S, Balasubramanian E, Karthik K, Chandrasekar U, Gupta Rishi (2015) Health monitoring of civil structures with integrated UAV and image processing system. Procedia Comput Sci 54:508-515

Sansoni G, Trebeschi M, Docchio F (2009) State-of-The-Art and Applications of 3D Imaging Sensors in Industry. Cult Herit Med Crim Investig Sens 9:568-601

Soldovieri F, Hugenschmidt J, Persico R, Leone G (2007) A linear inverse scattering algorithm for realistic GPR applications. Near Surf Geophys 5:29-42

Soldovieri F, Gennarelli G, Catapano I, Liao D, Dogaru T (2017) Forward-looking radar imaging: a comparison of two data processing strategies. IEEE J Sel Top Appl Earth Observ Remote Sens 10(2):562-571

Solimene R, Catapano I, Gennarelli G, Cuccaro A, Dell'Aversano A, Soldovieri F (2014) SAR imaging algorithms and some unconventional applications: a unified mathematical overview. IEEE Signal Process Mag 31(4):90-98

Solla M, Lorenzo H, Riveiro B, Rial FI (2011) Non-destructive methodologies in the assessment of the masonry arch bridge of Traba, Spain. Eng Fail Anal 18(3):828-835

Sternal M, Dragos K (2016) BIM-based modeling of Structural Health Monitoring systems using the IFC standard, Forum Bauinformatik 2016. Leibniz Universitat, Hannover

Ubertini F, Cavalagli N, Kita A, Comanducci G (2018) Assessment of a monumental masonry bell-tower after 2016 central Italy seismic sequence by long-term SHM. Bull Earthq Eng 16(2):775-801

Wang ML, Lynch JP, Sohn H (2014) Sensor technologies for civil infrastructures, applications in structural health monitoring, vol 2. Elsevier, Amsterdam

Williams J (2011) Applications for a switched-capacitor instrumentation building block. In: Dobkin B, Williams J (eds) Analog circuit design. Newnes, Oxford, pp 518-531

Zhang P (2010) Transducers and valves. In: Advanced industrial control technology. William Andrew Publishing, pp 117-152

Zumbahlen H (2008) Sensors. In: Zumbahlen H (ed) Linear circuit design handbook. Newnes, Oxford, pp 193-243

Publisher's Note Springer Nature remains neutral with regard to jurisdictional claims in published maps and institutional affiliations. 